Reprod. Nutr. Dévelop., 1988, 28 Suppl. $\mathrm{n}^{\circ} 1,171-172$

\title{
Etude de la stéatose hépatique en début de lactation chez les vaches laitières consommant de l'ensilage d'herbe
}

\author{
A. MAZUR, S. BAZIN $\left({ }^{*}\right)$, Y. RAYSSIGUIER
}

Laboratoire des Maladies Métaboliques, IN.R.A., Theix, 63122 Ceyrat, France.

(*) I.T.E.B., 149, rue de Bercy, 75595 Paris Cedex 12.

Summary. A survey of the incidence of post-parturient fatty liver in high yielding dairy cows was carried on 74 cows from 34 dairy herds fed grass silage. Triglycerides determination in liver biopsies indicated that $20 \%$ of cows had a moderate or severe fatty liver between 5 and 21 day post-partum.

Les premières semaines après le vêlage constituent une période critique chez la vache laitière haute productrice. En effet une lipomobilisation intense peut être à l'origine de stéatose hépatique qui s'accompagne de troubles pathologiques variés (Reid et Roberts, 1983). En raison de son ingestion insuffisante et de la difficulté d'assurer une complémentation adéquate en aliment concentré l'utilisation de l'ensilage d'herbe pourrait conduire à l'accentuation des problèmes métaboliques du début de lactation. L'objectif de cette étude a été d'évaluer la fréquence de la stéatose hépatique chez les vaches laitières en début de lactation consommant de l'ensilage d'herbe.

Matériel et méthodes. Nous avons étudié 74 vaches laitières de production supérieure à $5500 \mathrm{~kg}$ de lait, prises au hasard dans 34 élevages différents de la région Auvergne. Ces animaux recevaient comme fourrage essentiellement de l'ensilage d'herbe. Sur chaque vache, nous avons effectué, après la distribution des aliments, une biopsie hépatique et une prise de sang, au cours de la période de 5 à 21 jours après le vêlage (Mazur et al., 1988). Dans les échantillons hépatiques le niveau d'infiltration lipidique a été déterminé par le dosage enzymatique des triglycérides tissulaires (Mazur et al., 1986). Les paramètres plasmatiques : bêta-hydroxybutyrate (BHB), glucose, acides gras libres ( $A G L)$, cholestérol total et cholestérol libre ont été dosés par voie enzymatique.

Résultats et discussion. La teneur moyenne en triglycérides hépatiques a été de $28,0 \pm 3,9$ (médiane de 14,5) pour des valeurs comprises entre 2 et $191 \mathrm{mg} / \mathrm{g}$ de poids frais (fig. 1). Les animaux ont été répartis en vaches normales $(<50 \mathrm{mg} / \mathrm{g}$ ), modérément stéatosiques (comprises entre 50 et $100 \mathrm{mg} / \mathrm{g}$ ) et sévèrement stéatosiques ( $>100 \mathrm{mg} / \mathrm{g}$ ) (Reid et Roberts, 1983). Selon cette classification, 10 vaches (15\%) ont présenté une stéatose modérée et 4 (5\%) une stéatose sévère. Le degré d'infiltration lipidique du foie était corrélé de façon positive avec les taux d'AGL et de BHB plasmatiques et de façon négative avec la glycémie (tabl. 1). 


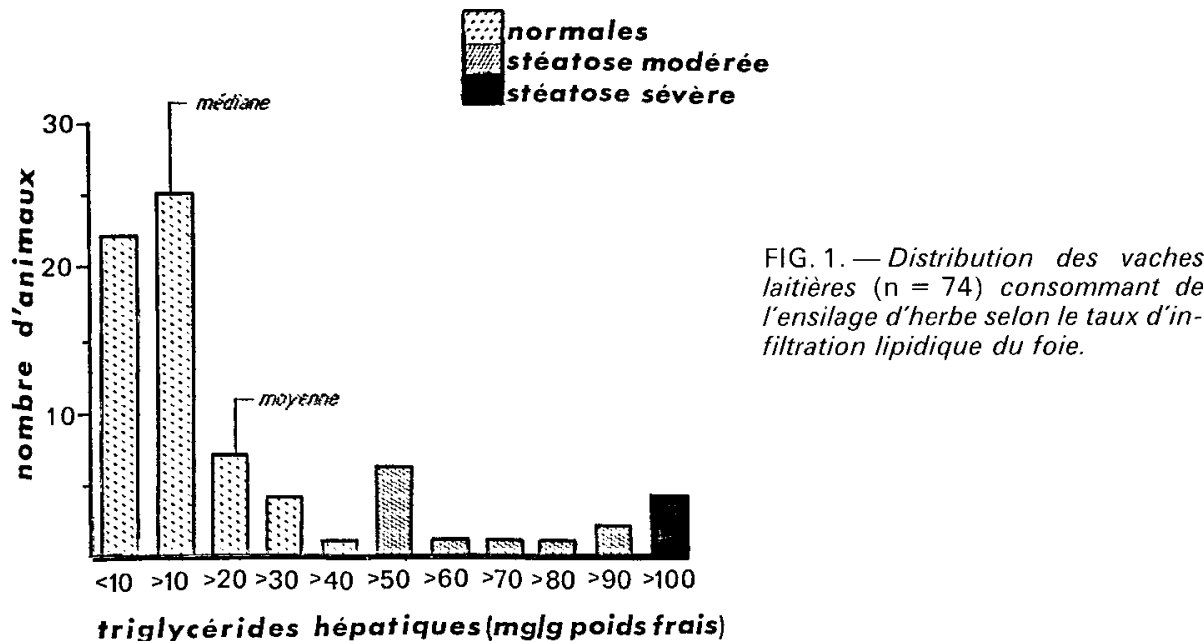

TABL. 1. - Concentration de certains métabolites plasmatiques chez les vaches laitières en début de factation consommant de l'ensilage d'herbe et leur relation avec l'infiltration lipidique du foie.

\begin{tabular}{llccr}
\hline & & $\begin{array}{c}\text { Vaches non stéatosiques } \\
(\mathrm{n}=60)\end{array}$ & $\begin{array}{c}\text { Vaches stéatosiques } \\
(\mathrm{n}=14)\end{array}$ & $\begin{array}{c}\text { Corrélation } \\
(\mathrm{n}=74)\end{array}$ \\
\hline Triglycérides hépatiques $(\mathrm{mg} / \mathrm{g})$ & \multicolumn{1}{c}{$\begin{array}{c} \\
(\mathrm{mM})\end{array}$} & $1,33 \pm 0,14$ & $1,89 \pm 0,26^{*}$ & $0,254^{*}$ \\
Bêta-hydroxybutyrate & $(\mathrm{mM})$ & $3,33 \pm 0,07$ & $3,00 \pm 0,12^{*}$ & $-0,263^{*}$ \\
Glucose & $(\mathrm{mM})$ & $0,37 \pm 0,03$ & $0,57 \pm 0,09^{* * *}$ & $0,393^{* *}$ \\
Acides gras libres & $(\mathrm{mg} / 100 \mathrm{ml})$ & $123,4 \pm 3,7$ & $117,4 \pm 6,1$ & $-0,031$ \\
Cholestérol total & $(\mathrm{mg} / 100 \mathrm{ml})$ & $26,2 \pm 0,8$ & $24,7 \pm 1,3$ & $-0,054$ \\
Cholestérol libre & &
\end{tabular}

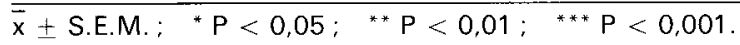

II faut donc souligner la fréquence relativement importante de la stéatose hépatique chez les animaux recevant un régime à base d'ensilage d'herbe (une vache sur 5 ). Le protocole adopté permettait davantage de faire un sondage sur l'existence de la stéatose chez les animaux recevant ce type d'alimentation, qu'une étude sur les facteurs de son apparition. Des analyses ultérieures des données zootechniques devraient permettre d'étudier les relations éventuelles entre le degré d'infiltration lipidique du foie et les caractéristiques des animaux, les rations alimentaires et la pathologie associée.

Mazur A., Bauchart D., Chilliard Y., Didier R., Rayssiguier Y., 1986. Reprod. Nutr. Dévelop., 26. 355-356.

Mazur A., Gueux E., Chilliard Y., Rayssiguier Y., 1988. J. anim. Physiol anim. Nutr., 59, 233-237. Reid I. M., Roberts C. J., 1983. Ir. Vet. J., 37, 104-110. 